\title{
Carboplatin-Cyclophosphamide or Paclitaxel without or with Bevacizumab as First-Line Treatment for Metastatic Triple-Negative Breast Cancer (BOOG 2013-01)
}

\author{
Annelot G.J. van Rossum ${ }^{\mathrm{a}}$ Ingrid A.M. Mandjes ${ }^{\mathrm{b}}$ Erik van Werkhoven ${ }^{\mathrm{c}}$ \\ Harm van Tinteren $^{c}$ A. Elise van Leeuwen-Stok ${ }^{d}$ Petra Nederlof $^{e}$ \\ Johanna E.A. Portielje ${ }^{f, g}$ Robbert J. van Alphen ${ }^{\text {h }}$ Els Platte ${ }^{i}$ \\ Daan van den Broek ${ }^{i}$ Alwin Huitema ${ }^{j}$ Marleen Kok ${ }^{k, l}$ Sabine C. Linn ${ }^{\text {a, l, } m}$ \\ Hendrika M. Oosterkamp ${ }^{n}$ on behalf of the Triple-B trialists' group \\ ${ }^{a}$ Division of Molecular Pathology, Netherlands Cancer Institute, Amsterdam, The Netherlands; ${ }^{\mathrm{b}}$ Data Center,

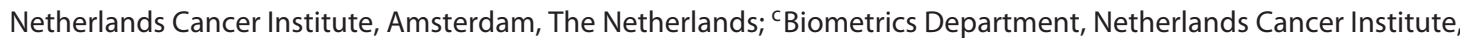 \\ Amsterdam, The Netherlands; ${ }^{\mathrm{d}}$ Dutch Breast Cancer Research Group, BOOG Study Center, Amsterdam, \\ The Netherlands; ${ }^{e}$ Department of Molecular Diagnostics, Netherlands Cancer Institute, Amsterdam, \\ The Netherlands; ${ }^{f}$ Department of Medical Oncology, HagaZiekenhuis, The Hague, The Netherlands; ${ }^{9}$ Department of

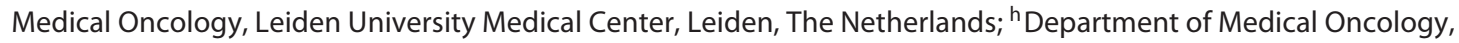 \\ Elisabeth-TweeSteden Hospital, Tilburg, The Netherlands; ${ }^{i}$ Clinical Chemical Laboratory, Netherlands Cancer

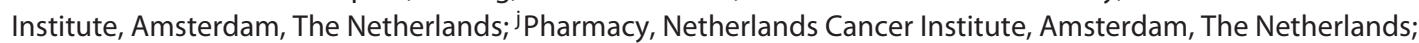 \\ kDivision of Molecular Oncology and Immunology, Netherlands Cancer Institute, Amsterdam, The Netherlands; \\ 'Department of Medical Oncology, Netherlands Cancer Institute, Amsterdam, The Netherlands; ${ }^{m}$ Department of \\ Pathology, University Medical Center, Utrecht, The Netherlands; ${ }^{n}$ Department of Medical Oncology, Haaglanden \\ Medisch Centrum, The Hague, The Netherlands
}

\section{Keywords}

Metastatic triple-negative breast cancer - Carboplatincyclophosphamide $\cdot$ Paclitaxel $\cdot$ Bevacizumab - Biomarker trial · Plasma vascular endothelial growth factor receptor-2

\footnotetext{
Abstract

Background: The addition of bevacizumab to chemotherapy conferred a modest progression-free survival (PFS) benefit in metastatic triple-negative breast cancer (mTNBC). However, no overall survival (OS) benefit has been reported. Also, its combination with carboplatin-cyclophosphamide (CC) has never been investigated. Methods: The Triple-B study is a multicenter, randomized phase IIb trial that aims to prospectively validate predictive biomarkers, including baseline plasma vascular endothelial growth factor recep-
}

tor-2 (pVEGFR-2), for bevacizumab benefit. mTNBC patients were randomized between $C C$ and paclitaxel $(P)$ without or with bevacizumab $(C C \pm B$ or $P \pm B)$. Here we report on a preplanned safety and preliminary efficacy analysis after the first 12 patients had been treated with $\mathrm{CC}+\mathrm{B}$ and on the predictive value of pVEGFR-2. Results: In 58 patients, the median follow-up was 22.1 months. Toxicity was manageable and consistent with what was known for each agent separately. There was a trend toward a prolonged PFS with bevacizumab compared to chemotherapy only (7.0 vs. 5.2 months; adjusted $\mathrm{HR}=0.60 ; 95 \% \mathrm{Cl} 0.33-1.08 ; p=0.09)$, but there was no effect on OS. In this small study, pVEGFR-2 concentration did not predict a bevacizumab PFS benefit. Both the intention-to-treat analysis and the per-protocol analysis did not

S.C.L. and H.M.O. contributed equally to this work. 
yield a significant treatment-by-biomarker test for interaction $\left(p_{\text {interaction }}=0.69\right)$. Conclusions: $C C$ and $C C+B$ are safe first-line regimens for $\mathrm{MTNBC}$ and the side effects are consistent with those known for each individual agent. pVEGFR-2 concentration did not predict a bevacizumab PFS benefit.

(c) 2021 S. Karger AG, Basel

\section{Introduction}

Triple-negative breast cancer (TNBC) accounts for $10-15 \%$ of all breast cancers and has a particularly poor prognosis [1]. The time from diagnosis to distant recurrences is shorter than for other breast cancer subtypes. Also, the median survival of patients with metastatic TNBC (mTNBC) is on average only 1 year [2] While conventional cytotoxic chemotherapy is still the backbone of treatment, new treatment strategies (i.e., targeted therapies and immune checkpoint blockers) are steadily making their way to the clinic [3-5]. However, our understanding of biological features of TNBC $[6,7]$ and potential treatment combinations is still inadequate. For example, there are indications that homologous recombination-deficient TNBC [8] is more sensitive to bifunctional alkylating and platinum agents than non-homologous recombination-deficient TNBC $[9,10]$, and that it is relatively resistant to taxanes [11]. However, whether it accounts for all of bifunctional alkylating and platinum agents is unknown.

Angiogenesis is important for tumor growth and development, particularly in TNBC. The expression of angiogenesis mediator vascular endothelial growth factor-A (VEGF-A) was found to be higher in TNBC than in nonTNBC [12, 13]. Therefore, inhibition of angiogenesis might be a potentially effective therapeutic target in this particular subtype [14]. The first results of bevacizumab, a monoclonal antibody against VEGF-A, in combination with chemotherapy were promising [15]. However, others found less pronounced treatment effects [16-18] and no overall survival (OS) benefit was seen in either of these trials. These modest results led to a search for biomarkers of bevacizumab benefit. Significant associations between the plasma VEGF-A (pVEGF-A) concentration and the survival benefit of bevacizumab were observed in retrospective analyses of breast cancer trials $[18,19]$. The MERiDiAN trial prospectively evaluated pVEGF-A.

Baseline plasma VEGFR-2 (pVEGFR-2) concentration was identified as another potential predictive biomarker for bevacizumab benefit in retrospective analyses [19]. The Triple-B trial aimed to prospectively analyze the predictive potential of baseline pVEGFR-2 concentration for bevacizumab efficacy. A coprimary objective was to validate the BRCA1-like classifier as a biomarker predictive of the efficacy of alkylating chemotherapy and plati- num compounds. As first-line therapy, mTNBC patients were treated with either carboplatin-cyclophosphamide or paclitaxel with or without bevacizumab $(\mathrm{CC} \pm \mathrm{B}$ and $\mathrm{P}$ $\pm \mathrm{B}$ respectively). Since $\mathrm{CC}$ had never been combined with bevacizumab before, a safety interim analysis was performed after 12 patients had been randomized in the $\mathrm{CC}+\mathrm{B}$ arm.

With the emerging evidence of only a modest bevacizumab efficacy and the results of the MERiDiAN trial demonstrating a limited utility of baseline pVEGFR-2 concentration as a biomarker predictive of bevacizumab efficacy, we deemed it necessary to adapt the Triple-B design and replace add-on bevacizumab with a different add-on. Therefore, we also report on the preliminary efficacy of bevacizumab addition.

\section{Methods}

\section{Patients}

The Triple-B study ("Biomarker Discovery Randomised Phase IIb Trial with Carboplatin-Cyclophosphamide versus Paclitaxel with or without Bevacizumab as First-Line Treatment in Advanced Triple-Negative Breast Cancer"; NCT01898117) is a randomized, multicenter, open-label, phase $2 \mathrm{~b}$ trial. Patients with histologically confirmed locally advanced TNBC or mTNBC were eligible. ER was considered negative when $<10 \%$ of the tumor cells showed nuclear staining. The tumor was negative for HER2 when immunohistochemical staining was 0 or $1+$ in intensity. In equivocal cases $(2+)$, an in situ hybridization assay was performed to determine the HER2 amplification status. Further eligibility criteria are listed in the online supplementary material (for all online suppl. material, see www.karger.com/doi/10.1159/000512200).

The REMARK (Reporting Recommendations for Tumor Marker Prognostic Studies) criteria were used to report this study [20].

\section{Treatment}

The patients were randomized among 4 treatment arms, i.e., (1) carboplatin area under curve (AUC) 5 and cyclophosphamide at $600 \mathrm{mg} / \mathrm{m}^{2}$ on day 1 every 4 weeks (CC), (2) carboplatin AUC 5 and cyclophosphamide at $600 \mathrm{mg} / \mathrm{m}^{2}$ on day 1 and bevacizumab at $10 \mathrm{mg} / \mathrm{kg}$ on days 1 and 15 every 4 weeks $(\mathrm{CC}+\mathrm{B}),(3)$ paclitaxel at $90 \mathrm{mg} / \mathrm{m}^{2}$ on days 1,8 , and 15 every 4 weeks (P), and (4) paclitaxel at $90 \mathrm{mg} / \mathrm{m}^{2}$ on days 1,8 , and 15 and bevacizumab at 10 $\mathrm{mg} / \mathrm{kg}$ on days 1 and 15 every 4 weeks $(\mathrm{P}+\mathrm{B})$. Treatment continued until progressive disease as defined by Response Evaluation Criteria In Solid Tumors (RECIST) version 1.1 [21] or unacceptable toxicity or upon the patient's request. In the case of an ongoing response and good tolerance in terms of toxicity after 6 cycles, patients and their treating physician were allowed to choose to either continue or stop treatment with chemotherapy and/or bevacizumab. Stratification factors were: (neo)adjuvant systemic treatment (yes vs. no), (neo)adjuvant taxane treatment (yes vs. no), and treatment center.

\section{Design and Objectives}

The Triple-B study was designed as a marker-by-treatment interaction trial with 2 primary objectives. The first primary objective was to test whether the baseline pVEGFR-2 concentration could indicate which patients have a longer progressionfree survival (PFS) with the addition of bevacizumab to first-line 
Table 1. Patient characteristics

\begin{tabular}{|c|c|c|c|c|}
\hline & $\mathrm{CC}(n=13)$ & $\mathrm{CC}+\mathrm{B}(n=15)$ & $\mathrm{P}(n=15)$ & $\mathrm{P}+\mathrm{B}(n=15)$ \\
\hline Age, years & $59(51-65)$ & $55(52-66)$ & $51(46-60)$ & $50(46-58.5)$ \\
\hline \multicolumn{5}{|l|}{ Surgery } \\
\hline None & $2(15.3)$ & $2(13.3)$ & 0 & $1(6.7)$ \\
\hline BCS & $5(38.5)$ & $7(46.7)$ & $5(33.3)$ & $1(6.7)$ \\
\hline Mastectomy & $6(46.2)$ & $6(40.0)$ & $10(66.7)$ & $13(86.7)$ \\
\hline \multicolumn{5}{|c|}{ Previous (neo)adjuvant chemotherapy } \\
\hline No & $2(15.4)$ & $4(26.7)$ & $2(13.3)$ & $4(26.7)$ \\
\hline Yes & $11(84.6)$ & $11(73.3)$ & $13(86.7)$ & $11(73.3)$ \\
\hline \multicolumn{5}{|c|}{ Previous (neo)adjuvant taxanes } \\
\hline No & $4(30.8)$ & $7(46.7)$ & $5(33.3)$ & $6(40.0)$ \\
\hline Yes & $9(69.2)$ & $8(53.3)$ & $10(66.7)$ & $9(60.0)$ \\
\hline \multicolumn{5}{|l|}{ DFS (months) } \\
\hline$\leq 24$ & $4(30.8)$ & $4(26.7)$ & $4(26.7)$ & $10(66.7)$ \\
\hline$>24$ & $9(69.2)$ & $11(73.3)$ & $11(73.3)$ & $5(33.3)$ \\
\hline \multicolumn{5}{|l|}{ Metastatic sites $(n)$} \\
\hline$\geq 3$ & $9(69.2)$ & $9(60.0)$ & $7(46.7)$ & $5(33.3)$ \\
\hline$<3$ & $4(30.8)$ & $6(40.0)$ & $8(53.3)$ & $10(66.7)$ \\
\hline \multicolumn{5}{|c|}{ Localization of disease } \\
\hline Locoregional & $1(7.7)$ & $1(6.7)$ & $1(6.7)$ & $3(20.0)$ \\
\hline Bone only & $2(15.4)$ & $3(20.0)$ & $2(13.3)$ & $4(26.7)$ \\
\hline Visceral & $3(23.1)$ & $3(20.0)$ & $4(26.7)$ & $3(20.0)$ \\
\hline Mixed & $7(53.8)$ & $8(53.3)$ & $8(53.3)$ & $5(33.3)$ \\
\hline \multicolumn{5}{|l|}{ Disease evaluation } \\
\hline Measurable & $11(84.6)$ & $14(93.3)$ & $11(73.3)$ & $9(60.0)$ \\
\hline Nonmeasurable & $2(15.4)$ & $1(6.7)$ & $4(26.7)$ & $6(40.0)$ \\
\hline \multicolumn{5}{|l|}{ pVEGFR-2 level $^{1}$} \\
\hline Low & $2(15.4)$ & $3(20.0)$ & $4(26.7)$ & $5(33.3)$ \\
\hline High & $10(76.9)$ & $9(60.0)$ & $10(66.7)$ & $6(40.0)$ \\
\hline Missing & $1(7.7)$ & $3(20.0)$ & $1(6.7)$ & $4(26.7)$ \\
\hline
\end{tabular}

Values are presented as medians (IQR) or numbers (\%).BCS, breast-conserving surgery. ${ }^{1}$ Split by prespecified cut-off into a low-VEGFR-2 ( $\leq 7.15 \mathrm{ng} / \mathrm{mL})$ subgroup and a high-VEGFR-2 $(>7.15 \mathrm{ng} / \mathrm{mL})$ subgroup.

chemotherapy for TNBC. PFS was defined as the time from randomization until progressive disease or death due to any cause, whichever occurred first. The other primary objective of the trial was to validate the $B R C A 1$-like profile as a predictive marker for the PFS benefit of CC compared with paclitaxel. Because validation of the BRCA1-like profile as a predictive marker is still a primary objective in the ongoing trial, it will be discussed in later reports.

The secondary endpoint OS was defined as the time from randomization until death by any cause. Toxicity was scored using the Common Toxicity Criteria for Adverse Events (CTCAE) version 4.03 .

Here we report on a planned interim analysis on safety after 12 patients were randomized to the $\mathrm{CC}+\mathrm{B}$ arm (there was a total of 58 patients in the entire cohort). With the emerging evidence of only a modest bevacizumab efficacy and the previously reported limited utility of baseline pVEGFR-2 concentration as a biomarker predictive of bevacizumab efficacy, we adapted the Triple-B design and replaced add-on bevacizumab with a different add-on, closing off the bevacizumab part of the trial. Therefore, we also report on the primary objective regarding the predictive value of the baseline pVEGFR-2 concentration and the (preliminary) efficacy of bevacizumab addition.

\section{pVEGFR-2 Concentration}

The pVEGFR-2 concentration was measured at baseline using a Quantikine Human VEGFR-2/KDR enzyme-linked immunosorbent assay (ELISA) kit (R\&D Systems, Minneapolis, MN, USA). The cohort was split, based on a prespecified cut-off derived from the AVADO trial [19], into a low-pVEGFR-2 subgroup ( $\leq 7.15 \mathrm{ng} /$ $\mathrm{mL}$ ) and a high-pVEGFR-2 subgroup (>7.15 $\mathrm{ng} / \mathrm{mL})$. Details on the measurement of pVEGFR-2 are provided in the online supplementary material.

\section{Statistics}

Details on the sample size calculation are provided in the online supplementary material.

Clinicopathologic characteristics of the 4 treatment groups were compared using the Kruskal-Wallis test, a $\chi^{2}$ test, or the Fisher exact test.

Efficacy was assessed in the intention-to-treat (ITT) population. The ITT population consisted of all patients who were allocated to one of the treatments. PFS and OS were estimated using the Kaplan-Meier method and compared using the log-rank test. Associations between clinicopathologic variables and survival endpoints were tested in univariable Cox regression models. If the Wald $p$ value was smaller than 0.2 , a variable was included in a multivariable Cox regression model. Multivariable Cox regression models were constructed to derive adjusted HR. 


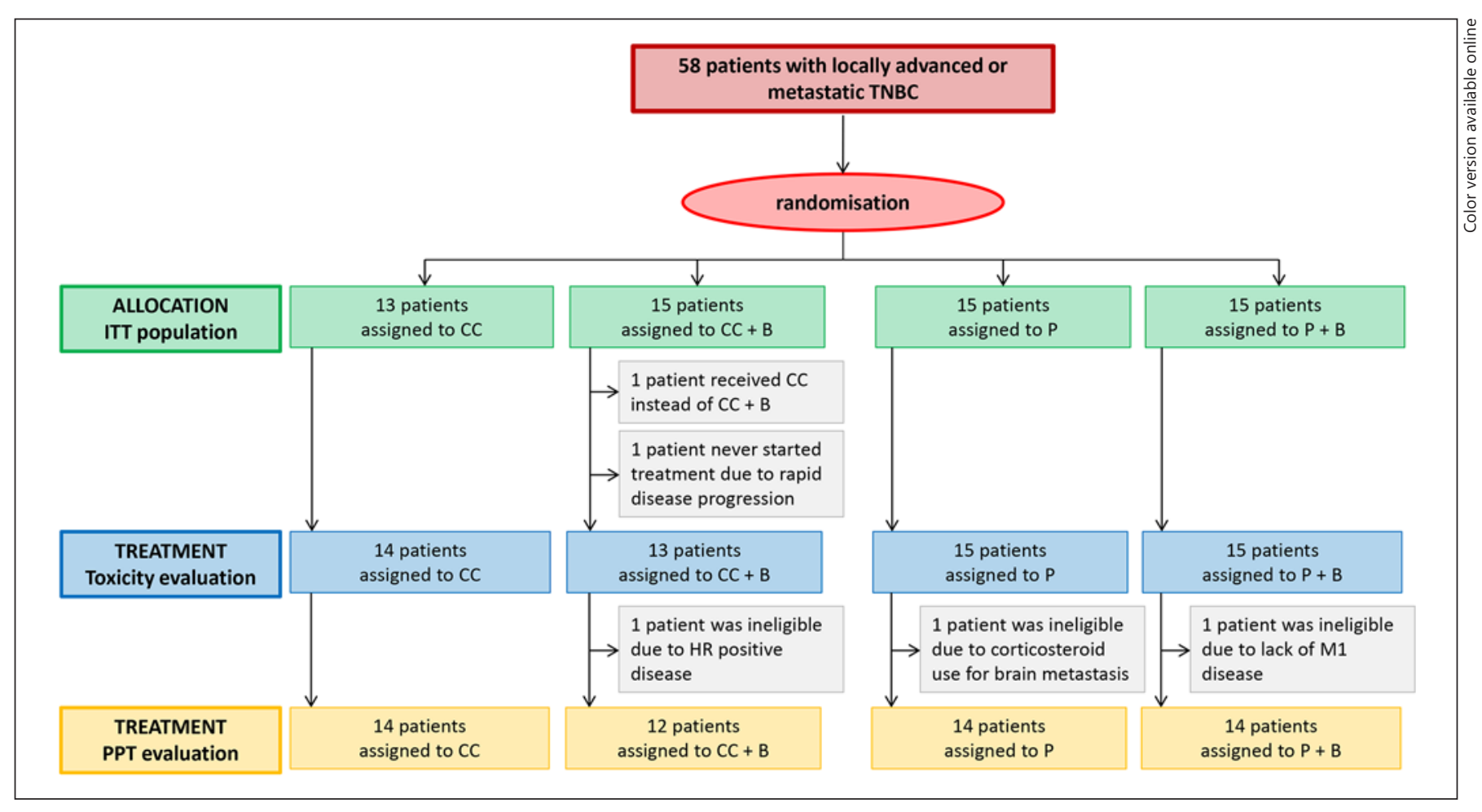

Fig. 1. CONSORT diagram.

The association between the baseline pVEGFR-2 concentration and PFS was assessed using the Kaplan-Meier method and compared using a log-rank test in the ITT population and the per protocol treatment (PPT) population. The PPT population included all eligible patients who received at least 1 cycle of the allocated treatment. Cox regression models were built to assess the hazard and to test for interaction.

All patients who received at least 1 cycle of the allocated treatment were evaluated for toxicity. Adverse events (AE) and serious AE (SAE) were described per treatment arm.

Two-sided $p$ values $<0.05$ were considered statistically significant. Analyses were performed using R software (version 3.3.1).

\section{Results}

From October 2013 to January 2018, fifty-eight patients were enrolled in 22 centers in The Netherlands (Table 1). Baseline characteristics were balanced between the treatment groups. The majority of the patients (46 out of 58 patients; $79.3 \%$ ) received (neo)adjuvant taxane-containing chemotherapy. Only 2 patients, i.e., 1 in the CC arm and 1 in the $\mathrm{P}+\mathrm{B}$ arm, were treated with carboplatin. Also, 36 patients [62\%] had a distant recurrence-free interval of more than 24 months. Figure 1 shows the number of patients included in the analyses for toxicity $(n=57)$, ITT $(n=58)$, and PPT $(n=54)$.

\section{Adverse Events}

The most common grade 2 or higher AEs are listed in Table 2. As expected, we observed more AEs in the bevacizumab-containing treatment arms $(\mathrm{CC}+\mathrm{B}$ or $\mathrm{P}+\mathrm{B})$ than in the chemotherapy-only arms (CC or P; online suppl. Table 1). Hypertension (11 out of 28 [39.3\%] vs. 2 out of 29 patients [6.9\%]; $p<0.01)$ and fatigue $(11[39.3 \%]$ vs. 4 patients $[13.8 \%] ; p=0.04)$ were observed more frequently in the bevacizumab-containing treatment arms compared to the chemotherapy-only treatment arms.

Anemia ( 11 out of 27 [40.7\%] vs. 2 out of 30 patients [6.7\%]; $p<0.01$ ), nausea (7 [25.9\%] vs. 0 patients; $p<$ $0.01)$, and vomiting (7 [25.9\%] vs. 0 patients; $p<0.01$ ) were more frequent in the $\mathrm{CC} \pm \mathrm{B}$ arms compared to the $\mathrm{P} \pm \mathrm{B}$ arms (online suppl. Table 2). In contrast, alopecia $(0$ vs. 6 patients $[20.0 \%] ; p=0.03)$ and peripheral neuropathy $(2[7.4 \%]$ vs. 10 patients [33.3\%]; $p=0.02)$ were more common AEs in the $\mathrm{P} \pm \mathrm{B}$ arms.

\section{Serious Adverse Events}

All possibly related SAEs are listed in Table 3. The most common SAEs were fever (3 patients; 5.2\%), anemia (2 patients; $3.5 \%$ ), and diarrhea (2 patients; $3.5 \%)$. Four CC + B-treated patients [28.6\%] encountered at least 1 SAE, compared to 2 patients in the CC arm [14.3\%] and the $\mathrm{P}+\mathrm{B}$ arm [13.3\%], respectively, and none in the subgroup of patients treated with P. No major bevacizumabrelated SAEs were observed. 
Table 2. AE per treatment arm, at least possibly related to the study treatment and observed in at least $10 \%$ of all of the patients

\begin{tabular}{|c|c|c|c|c|c|c|c|c|}
\hline & \multicolumn{2}{|c|}{$\mathrm{CC}(n=14)$} & \multicolumn{2}{|c|}{$\mathrm{CC}+\mathrm{B}(n=13)$} & \multicolumn{2}{|c|}{$\mathrm{P}(n=15)$} & \multicolumn{2}{|c|}{$\mathrm{P}+\mathrm{B}(n=15)$} \\
\hline & grade 2 & grade $3-4$ & grade 2 & grade $3-4$ & grade 2 & grade $3-4$ & grade 2 & grade $3-4$ \\
\hline Alopecia & 0 & 0 & 0 & 0 & $3(20.0)$ & 0 & $3(20.0)$ & 0 \\
\hline Anemia & $6(42.9)$ & 0 & $3(21.4)$ & $2(14.3)$ & 0 & 0 & $2(13.3)$ & 0 \\
\hline Fatigue & $3(21.4)$ & 0 & $3(21.4)$ & $3(21.4)$ & $1(6.7)$ & 0 & $5(33.3)$ & 0 \\
\hline Hypertension & $1(7.1)$ & 0 & $1(7.1)$ & $5(35.7)$ & 0 & $1(6.7)$ & $1(6.7)$ & $4(26.7)$ \\
\hline Nausea & $2(14.3)$ & $1(7.1)$ & $4(28.6)$ & 0 & 0 & 0 & 0 & 0 \\
\hline Decreased neutrophil count & 0 & $6(42.9)$ & $3(21.4)$ & $8(61.5)$ & $5(33.3)$ & $3(20.0)$ & $5(33.3)$ & $2(13.3)$ \\
\hline Peripheral sensory neuropathy & 0 & 0 & $2(14.3)$ & 0 & $5(33.3)$ & 0 & $3(20.0)$ & $1(6.7)$ \\
\hline Vomiting & $2(14.3)$ & 0 & $5(35.7)$ & 0 & 0 & 0 & 0 & 0 \\
\hline Decreased white blood cells & $1(7.1)$ & $6(42.9)$ & $4(28.6)$ & $5(38.5)$ & $5(33.3)$ & $1(6.7)$ & $3(20.0)$ & $1(6.7)$ \\
\hline Total AE & 24 & 19 & 42 & 29 & 24 & 8 & 35 & 15 \\
\hline
\end{tabular}

Values are presented as numbers (\%).

Table 3. SAE at least possibly related to the study treatment

\begin{tabular}{lllll}
\hline & $\mathrm{CC}(n=14)$ & $\mathrm{CC}+\mathrm{B}(n=13)$ & $\mathrm{P}(n=15)$ & $\mathrm{P}+\mathrm{B}(n=15)$ \\
\hline $\begin{array}{l}\text { Anaemia } \\
\text { Diarrhoea }\end{array}$ & $2(14.3)$ & \\
$\begin{array}{l}\text { Fever } \\
\text { Decreased neutrophil count }\end{array}$ & $1(7.1)$ & $2(14.3)$ & $2(13.3)$ \\
$\begin{array}{l}\text { Decreased platelet count } \\
\text { Thromboembolic event }\end{array}$ & $1(7.1)$ & $1(7.1)$ & \\
$\begin{array}{l}\text { Decreased white blood cells } \\
\text { Total patients with } \geq 1 \text { SAE }\end{array}$ & $2(14.3)$ & $4(28.6)$ & $0(0.0)$ & $2(13.3)$ \\
\hline
\end{tabular}

Values are presented as numbers $(\%)$

\section{Treatment Exposure}

Patients received on average 4-6 cycles of treatment and 2 additional cycles of bevacizumab (single agent) in the CC + B-treated arm (online suppl. Table 3 ). Dose reductions and delays occurred more often in the treatment arms with bevacizumab $(\mathrm{CC}+\mathrm{B}$ and $\mathrm{P}+\mathrm{B})$ than in the chemotherapy-only arms (CC and $\mathrm{P})$. The relative total dose intensity of patients who were treated with $\mathrm{CC}+\mathrm{B}$ was lower than that of the CC-treated subgroup (89.6 vs. 96.9\%; Student $t$ test $p=0.04$ ). Four out of 57 patients (7\%) discontinued treatment due to toxicity, i.e., 1 due to bone marrow toxicity, 1 due to peripheral sensory neuropathy, 1 due to ongoing pruritus, and 1 due to multiple side effects.

\section{Efficacy}

The median follow-up was 22.1 months (IQR 18.430.4). Patients treated with bevacizumab (CC + B or P + B) had a significantly longer PFS than the chemotherapyonly subgroup (CC or P; median PFS: 7.0 vs. 5.2 months; unadjusted $\mathrm{HR}=0.56 ; 95 \%$ CI $0.32-0.98 ; p=0.04 ;$ Fig. $2 \mathrm{a}$ ).
Age and use of neoadjuvant or adjuvant chemotherapy were significantly associated with PFS in the univariable analysis. Corrected for these factors, PFS was not significantly different between bevacizumab-treated subgroups and chemotherapy-only subgroups (adjusted HR =0.60; 95\% CI 0.33-1.08; $p=0.09$ ).

Split by chemotherapy backbone, PFS was significantly longer for $\mathrm{CC}+\mathrm{B}$-treated patients than for CC-treated patients (median PFS: 7.0 vs. 4.3 months; unadjusted HR $=0.39 ; 95 \%$ CI $0.16-0.95 ; p=0.04$; Fig. 2b). However, the difference was no longer significant when corrected for prognostic factors (adjusted $\mathrm{HR}=0.45 ; 95 \%$ CI $0.18-1.10$; $p=0.08)$. We did not observe a significant difference in PFS between the $\mathrm{P}+\mathrm{B}$-treated subgroup and paclitaxeltreated patients (7.0 vs. 6.1 months; adjusted $\mathrm{HR}=0.74$; $95 \%$ CI $0.32-1.70 ; p=0.48$; Fig. 2c). An overview of the time to progression of all of the patients is given in online supplementary Figure 1.

OS was not significantly different for bevacizumabtreated patients $(\mathrm{CC}+\mathrm{B}$ or $\mathrm{P}+\mathrm{B})$ compared to the chemotherapy-only subgroup (CC or P; 17.7 vs. 15.4 months; 


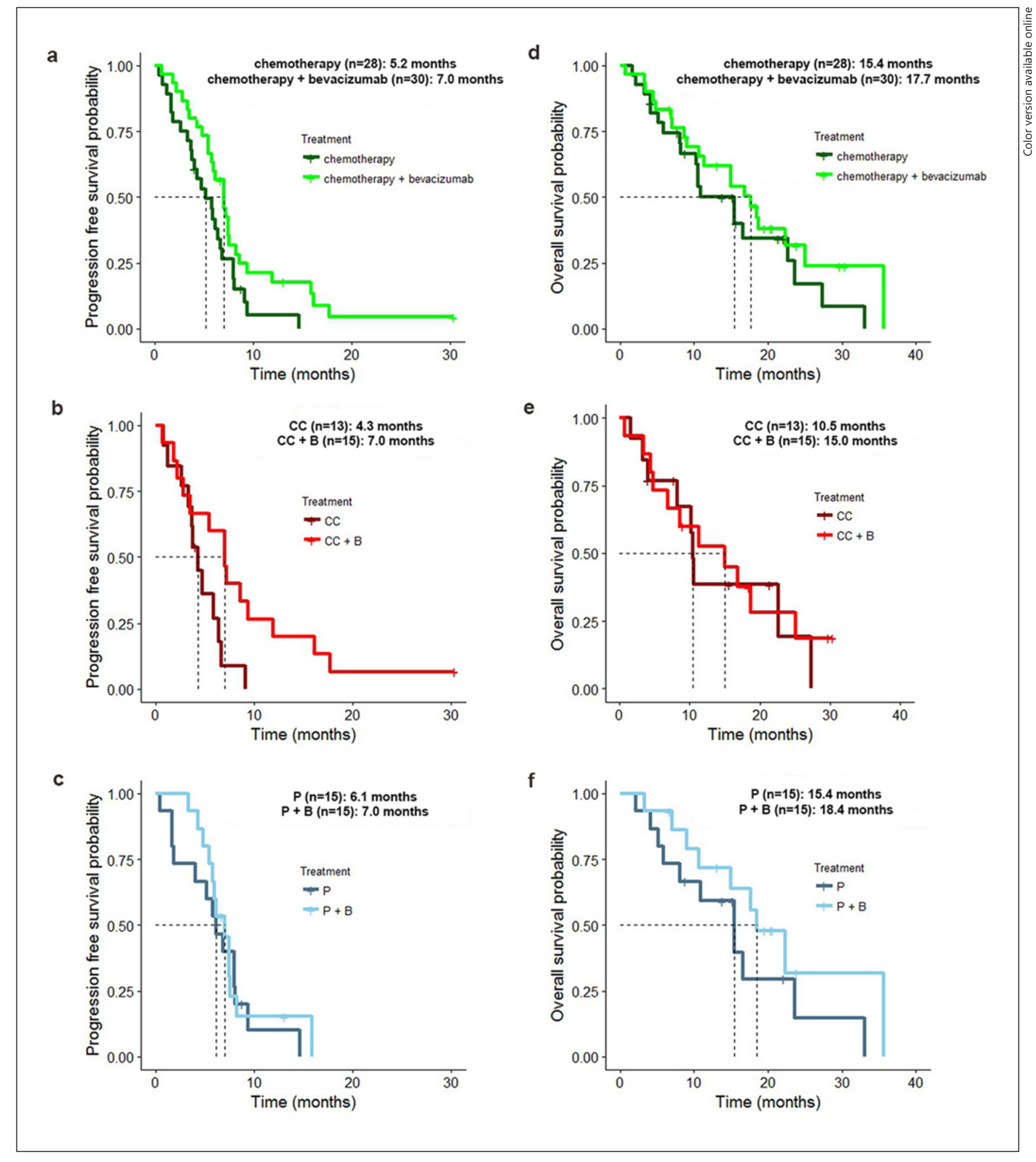

Fig. 2. Association between treatment and the PFS (a-c) and OS (d-f) of the ITT population.

Fig. 2d) when corrected for age, use of neoadjuvant or adjuvant chemotherapy, use of neoadjuvant or adjuvant taxanes, and the number of metastatic sites (adjusted HR $=0.88 ; 95 \%$ CI $0.46-1.69 ; p=0.70)$. Similarly, no signifi- cant difference in OS was observed when treatments were split by chemotherapy backbone ( $\mathrm{CC}$ vs. $\mathrm{CC}+\mathrm{B}$ and $\mathrm{P}$ vs. $\mathrm{P}+\mathrm{B}$; Fig. 2e, f). 


\section{Plasma VEGFR-2}

For 49 out of 58 ITT patients (84.5\%; Table 1), pVEGFR-2 concentrations were measured. In all of the patients, the baseline pVEGFR-2 concentration was not significantly associated with PFS (adjusted for age and use of neoadjuvant or adjuvant chemotherapy, $\mathrm{HR}=1.16$; 95\% CI 0.59-2.29; $p=0.67)$. Split by pVEGFR-2 concentration, patients with a low pVEGFR-2 concentration had a significantly longer PFS with bevacizumab treatment $(\mathrm{CC}+\mathrm{B}$ or $\mathrm{P}+\mathrm{B})$ compared to chemotherapy only $(\mathrm{CC}$ or P; median PFS: 7.0 vs. 3.5 months; adjusted $\mathrm{HR}=0.23$; 95\% CI 0.06-0.91; $p=0.04$; online suppl. Fig. 2a). However, PFS was not significantly different in patients with a high pVEGFR-2 concentration when treated with CC + $\mathrm{B}$ or $\mathrm{P}+\mathrm{B}$ compared to $\mathrm{CC}$ or paclitaxel (7.0 vs. 5.8 months; adjusted HR $=0.61 ; 95 \%$ CI $0.27-1.36 ; p=0.23$; online suppl. Fig. 2b). Also, the interaction between treatment (chemotherapy without or with bevacizumab [CC and $\mathrm{P}$ vs. $\mathrm{CC}+\mathrm{B}$ and $\mathrm{P}+\mathrm{B}]$ ) and pVEGFR-2 concentration was not significant $\left(p_{\text {interaction }}=0.69\right)$. The pVEGFR-2 analysis in the PPT population is displayed in online supplementary Figure $2 c$ and d (low-pVEGFR-2 subgroup: adjusted $\mathrm{HR}=0.38 ; 95 \%$ CI $0.15-0.94 ; p=0.04$; high-pVEGFR-2 subgroup: adjusted $\mathrm{HR}=0.26$; $95 \% \mathrm{CI}$ $0.06-1.09 ; p=0.07)$. Also in the PPT population, the test for interaction was not significant $\left(p_{\text {interaction }}=0.75\right)$.

\section{Discussion}

Here we report on the toxicity and efficacy of 2 chemotherapy backbones (CC and $\mathrm{P}$ ) without or with the addition of bevacizumab $(\mathrm{CC} \pm \mathrm{B}$ and $\mathrm{P} \pm \mathrm{B}$ ) as first-line treatment for $\mathrm{mTNBC}$ patients in the randomized Triple- $\mathrm{B}$ study. We showed that it is safe to add bevacizumab to CC and that toxicity is slightly different from that of $\mathrm{P}+\mathrm{B}$. The addition of bevacizumab to these chemotherapy regimens resulted in a trend toward a longer PFS. The OS was not significantly prolonged after bevacizumab-containing treatment.

The efficacy results of the addition of bevacizumab are in line with the hormone receptor-negative or TNBC subgroup analyses [22] of the E2100 trial [15], the AVADO trial [17], and the RIBBON-1 trial [16]. The AVADO trial and the RIBBON-1 trial showed that the addition of bevacizumab to chemotherapy prolonged PFS but not OS. A meta-analysis on HER-2-negative breast cancer [22] and a more recent trial on hormone receptor-positive breast cancer (bevacizumab combined with endocrine therapy) [23] showed the same. Although we can only speculate as to an explanation, it might be caused by selective inhibition of VEGF-A by bevacizumab. Since other isoforms of VEGF are still able to bind to their receptor on endothelial cells [24], blocking VEGF-A might result in a temporary effect on tumor progression. In time other isoforms might take over to stimulate angiogenesis and tumor growth, thereby impairing the efficacy of later lines of chemotherapy. Besides VEGF, other mediators might promote angiogenesis. [25] The recurrent finding of a PFS benefit but not an OS benefit of bevacizumab hampers its widespread use. It is highly likely that there is a subgroup of patients with an OS benefit of bevacizumab. However, a predictive biomarker for this particular group is still lacking.

To our knowledge, this is the first trial to randomize breast cancer patients to the combination of CC. Furthermore, this is the first time that bevacizumab is added to this combination. CC has previously been introduced as a safe and effective treatment for ovarian cancer [26-28]. In agreement with these reports, anemia, nausea, and vomiting occurred more often in the CC arms than in the paclitaxel arms. Although we did observe more toxicity when bevacizumab was added to CC, this combination was considered safe by the independent data safety-monitoring board.

The CC arms resulted in PFS and OS that were similar to those of the paclitaxel arms in our cohort, with PFS of 5.5 months for $\mathrm{CC} \pm \mathrm{B}$ and 6.5 months for $\mathrm{P} \pm \mathrm{B}$. Interestingly, 3 patients treated with $\mathrm{CC}+\mathrm{B}$ had a PFS of more than 16 months. Considering that the average OS of $\mathrm{mT}$ NBC patients is 1 year [2], these patients responded remarkably well to the treatment. Besides a shared high pVEGFR-2 concentration, we could not find an explanation based on patient or tumor characteristics at this point. The second primary objective of this trial was to validate the $B R C A 1$-like profile as a predictive biomarker for the survival benefit of alkylating agents and platinum compounds compared to taxanes. Planned future analyses of the BRCA1-like status of these tumors might shed new light on this matter.

We prospectively tested the baseline pVEGFR-2 concentration as a predictive biomarker for the PFS and OS benefit of the addition of bevacizumab to first-line chemotherapy for TNBC. Previously, 2 groups showed in a retrospective analysis that the baseline pVEGFR-2 concentration was a promising biomarker for the PFS benefit of bevacizumab in HER2-negative breast cancer patients [18, 19]. In our relatively small study, we could not confirm these earlier findings, suggesting a limited predictive value for baseline pVEGFR-2 concentration. Our findings are in line with the results of the MERiDiAN study [29] in which the interaction between pVEGFR-2 concentration and bevacizumab treatment was also not significant. When the pVEGFR-2 concentration cut-off used in the AVADO trial was applied, the majority of our patients had a high pVEGFR-2 concentration. The cut-off used in the MERiDiAN study was somewhat higher $(11.2 \mathrm{ng} / \mathrm{mL})$. Even when applying different cut-offs, including the concentration used 
in the MERiDiAN trial, the interaction between pVEGFR-2 concentration and PFS was not significant.

A major limitation of the analyses of this report is that the number of patients is lower than planned in advance. Designed as a marker-by-treatment interaction trial, sample size calculations were based on the anticipated treatment effect and the size of the biomarker subgroups. Due to low rate of accrual because of (1) emerging evidence that bevacizumab was less promising than expected and (2) the MERiDiAN trial results indicating that baseline pVEGFR-2 concentration was not a suitable biomarker for bevacizumab benefit, we were forced to amend the protocol and to bring the bevacizumab-part of the trial to a close. Therefore, the number of patients in these analyses is limited.

Summarizing, $C C \pm B$ is a safe first-line treatment for mTNBC. The difference in toxicity profile between CC and paclitaxel can be useful to guide treatment choices in the management of mTNBC. Although this result was obtained from a cohort that was stopped at interim and should therefore be interpreted with caution, bevacizumab addition to paclipaxel or CC prolonged the PFS. For OS, no benefit was observed. In this small cohort, we were not able to validate baseline pVEGFR-2 concentration as a predictive biomarker for bevacizumab benefit. Given the biological heterogeneity and variation in responses, predictive biomarkers for treatment efficacy in TNBC are needed. Marker-by-treatment interaction trials, such as the ongoing Triple-B study, are required to validate these biomarkers and consequently optimize treatment decisions.

\section{Acknowledgments}

First, we would like to thank all of the patients and their families. Also, we would like to express our gratitude to the Dutch Breast Cancer Research Group (BOOG), the study teams of the participating centers, and the Data Center, the Clinical Chemical Laboratory, and the Molecular Diagnostics of the Netherlands Cancer Institute. Finally, we would like to thank the members of the DSMB for their careful consideration.

\section{Statement of Ethics}

All of the patients gave written informed consent. This study was performed in accordance with the Declaration of Helsinki. The study protocol and its amendments were reviewed and approved by the Ethical Committee of the Netherlands Cancer Institute and the institutional boards of the participating centers.

\section{Disclosure statement}

S.C.L. is an advisory board member for Cergentis, IBM, Novartis, Pfizer, Roche, and Sanofi and received institutional research support funding from Amgen, AstraZeneca, Genentech, Roche, Sanofi, and TESARO. H.M.O. is an advisory board member for Roche, Pfizer, and Novartis and received institutional research support funding from Roche. M.K. is an advisory board member for BMS and received institutional research support funding from Roche and BMS. All of the other authors declare no potential conflict of interests.

\section{Funding Sources}

The Triple-B trial was funded by an unrestricted research grant from Roche to the legal sponsor BOOG. The funding source had no role in the design and conduction of this study; collection, management, analysis, and interpretation of the data; preparation or approval of this paper; or the decision to submit this paper for publication.

\section{Author Contributions}

S.C.L., H.M.O., H.T., and D.B. were involved in the study conception and design. S.C.L., H.M.O., I.A.M.M., A.G.J.R., P.N., J.E.A.P., R.J.A., and E.P performed data acquisition. I.A.M.M., E.W., D.B., P.N., A.G.J.R., M.K., S.C.L., and H.M.O. were responsible for data quality control, analysis, and interpretation. A.G.J.R. and E.W. performed the statistical analysis. A.G.J.R., M.K., S.C.L., and H.M.O prepared and edited this paper. All of the authors reviewed and approved this work.

\section{References}

1 Dent R, Trudeau M, Pritchard KI, Hanna WM, Kahn HK, Sawka CA, et al. Triple-negative breast cancer: clinical features and patterns of recurrence. Clin Cancer Res. 2007 Aug;13(15 Pt 1):4429-34

2 Li X, Yang J, Peng L, Sahin AA, Huo L, Ward $\mathrm{KC}$, et al. Triple-negative breast cancer has worse overall survival and cause-specific survival than non-triple-negative breast cancer. Breast Cancer Res Treat. 2017 Jan;161(2): 279-87.

3 Lee A, Djamgoz MB. Triple negative breast cancer: emerging therapeutic modalities and novel combination therapies. Cancer Treat Rev. 2018 Jan;62:110-22.
4 Schmid P, Adams S, Rugo HS, Schneeweiss A, Barrios $\mathrm{CH}$, Iwata $\mathrm{H}$, et al.; IMpassion 130 Trial Investigators. Atezolizumab and Nab-Paclitaxel in Advanced Triple-Negative Breast Cancer. N Engl J Med. 2018 Nov;379(22): 2108-21.

5 Vinayak S, Tolaney SM, Schwartzberg L, Mita M, McCann G, Tan AR, et al. Open-Label Clinical Trial of Niraparib Combined With Pembrolizumab for Treatment of Advanced or Metastatic Triple-Negative Breast Cancer. JAMA Oncol. 2019 Jun;5(8):1132.

6 Foulkes WD, Smith IE, Reis-Filho JS. Triplenegative breast cancer. N Engl J Med. 2010 Nov;363(20):1938-48.
7 Lehmann BD, Jovanović B, Chen X, Estrada MV, Johnson KN, Shyr Y, et al. Refinement of Triple-Negative Breast Cancer Molecular Subtypes: Implications for Neoadjuvant Chemotherapy Selection. PLoS One. 2016 Jun; 11(6):e0157368.

8 Severson TM, Peeters J, Majewski I, Michaut M, Bosma A, Schouten PC, et al. BRCA1-like signature in triple negative breast cancer: molecular and clinical characterization reveals subgroups with therapeutic potential. Mol Oncol. 2015 Oct;9(8):1528-38. 
9 Vollebergh MA, Lips EH, Nederlof PM, Wessels LF, Schmidt MK, van Beers EH, et al. An aCGH classifier derived from BRCA1-mutated breast cancer and benefit of high-dose platinum-based chemotherapy in HER2-negative breast cancer patients. Ann Oncol. 2011 Jul; 22(7):1561-70.

10 Schouten PC, Marme F, Aulmann S, Sinn HP van Essen $\mathrm{HF}$, Ylstra B, et al. Breast cancers with a BRCA1-like DNA copy number profile recur less often than expected after high-dose alkylating chemotherapy. Clin Cancer Res. 2015 Feb;21(4):763-70.

11 Akashi-Tanaka S, Watanabe C, Takamaru T, Kuwayama T, Ikeda M, Ohyama $\mathrm{H}$, et al. BRCAness predicts resistance to taxane-containing regimens in triple negative breast cancer during neoadjuvant chemotherapy. Clin Breast Cancer. 2015 Feb;15(1):80-5.

12 Linderholm BK, Hellborg H, Johansson U, Elmberger G, Skoog L, Lehtio J, et al. Significantly higher levels of vascular endothelial growth factor (VEGF) and shorter survival times for patients with primary operable triple-negative breast cancer. Ann Oncol. 2009 Oct;20(10):1639-46.

13 Bender RJ, Mac Gabhann F. Expression of VEGF and semaphorin genes define subgroups of triple negative breast cancer. PLoS One. 2013 May;8(5):e61788.

14 Ribatti D, Nico B, Ruggieri S, Tamma R, Simone G, Mangia A. Angiogenesis and Antiangiogenesis in Triple-Negative Breast cancer. Transl Oncol. 2016 Oct;9(5):453-7.

15 Miller K, Wang M, Gralow J, Dickler M, Cobleigh M, Perez EA, et al. Paclitaxel plus bevacizumab versus paclitaxel alone for metastatic breast cancer. N Engl J Med. 2007 Dec; 357(26):2666-76.

16 Robert NJ, Diéras V, Glaspy J, Brufsky AM, Bondarenko I, Lipatov ON, et al. RIBBON-1: randomized, double-blind, placebo-controlled, phase III trial of chemotherapy with or without bevacizumab for first-line treatment of human epidermal growth factor receptor 2-negative, locally recurrent or metastatic breast cancer. J Clin Oncol. 2011 Apr;29(10):1252-60.
17 Miles DW, Chan A, Dirix LY, Cortés J, Pivot $\mathrm{X}$, Tomczak $\mathrm{P}$, et al. Phase III study of bevacizumab plus docetaxel compared with placebo plus docetaxel for the first-line treatment of human epidermal growth factor receptor 2-negative metastatic breast cancer. J Clin Oncol. 2010 Jul;28(20):3239-47.

18 Cameron D, Brown J, Dent R, Jackisch C, Mackey J, Pivot X, et al. Adjuvant bevacizumab-containing therapy in triple-negative breast cancer (BEATRICE): primary results of a randomised, phase 3 trial. Lancet Oncol. 2013 Sep;14(10):933-42.

19 Miles DW, de Haas SL, Dirix LY, Romieu G, Chan A, Pivot X, et al. Biomarker results from the AVADO phase 3 trial of first-line bevacizumab plus docetaxel for HER2-negative metastatic breast cancer. Br J Cancer. 2013 Mar;108(5):1052-60.

20 Sauerbrei W, Taube SE, McShane LM, Cavenagh MM, Altman DG. Reporting Recommendations for Tumor Marker Prognostic Studies (REMARK): An Abridged Explanation and Elaboration. J Natl Cancer Inst. 2018 Aug;110(8):803-11.

21 Eisenhauer EA, Therasse P, Bogaerts J, Schwartz LH, Sargent D, Ford R, et al. New response evaluation criteria in solid tumours: revised RECIST guideline (version 1.1). Eur J Cancer. 2009 Jan;45(2):228-47.

22 Miles DW, Dieras V, Cortes J, Duenne AA, Yi J, O'Shaughnessy J. First-line bevacizumab in combination with chemotherapy for HER2negative metastatic breast cancer: pooled and subgroup analyses of data from 2447 patients. Ann Oncol. 2013 Nov;24(11):2773-80.

23 Martín M, Loibl S, Hyslop T, De la HabaRodríguez J, Aktas B, Cirrincione CT, et al.; GEICAM Spanish Breast Cancer Group; GBG (German Breast Group); Alliance for Clinical Trials in Oncology (Alliance). Evaluating the addition of bevacizumab to endocrine therapy as first-line treatment for hormone receptor-positive metastatic breast cancer: a pooled analysis from the LEA (GEICAM/2006-11 GBG51) and CALGB 40503 (Alliance) trials. Eur J Cancer. 2019 Aug;117:91-8.
24 Stevenson CE, Nagahashi M, Ramachandran S, Yamada A, Bear HD, Takabe K. Bevacizumab and breast cancer: what does the future hold? Future Oncol. 2012 Apr;8(4):40314.

25 Mackey JR, Kerbel RS, Gelmon KA, McLeod DM, Chia SK, Rayson D, et al. Controlling angiogenesis in breast cancer: a systematic review of anti-angiogenic trials. Cancer Treat Rev. 2012 Oct;38(6):673-88.

26 Tredan O, Geay JF, Touzet S, Delva R, Weber B, Cretin J, et al. Carboplatin/cyclophosphamide or carboplatin/paclitaxel in elderly patients with advanced ovarian cancer? Analysis of two consecutive trials from the Groupe d'Investigateurs Nationaux pour l'Etude des Cancers Ovariens. Ann Oncol. 2007 Feb; 18(2):256-62.

27 Meerpohl HG, Sauerbrei W, Kühnle H, Schumacher M, Pfleiderer A; German Ovarian Cancer Study Group (GOCA). Randomized study comparing carboplatin/cyclophosphamide and cisplatin/cyclophosphamide as first-line treatment in patients with stage III/ IV epithelial ovarian cancer and small volume disease. Gynecol Oncol. 1997 Jul;66(1):75-84

28 Edmonson JH, Suman VJ, Dalton RJ, Bro WC, Gallenberg MM, Long HJ, et al.; North Central Cancer Treatment Group. Comparison of conventional dose and double dose carboplatin in patients receiving cyclophosphamide plus carboplatin for advanced ovarian carcinoma: a North Central Cancer Treatment Group Study. Cancer Invest. 2001 19(6):597-602.

29 Das Thakur M, Bais C, Estay I, Vaidyanathan $\mathrm{R}$, O'Shaughnessy J, Cameron D, et al. Biomarker results from MERiDiAN, a doubleblind placebo-controlled randomized phase III trial of first-line paclitaxel with or without bevacizumab for HER2-negative metastatic breast cancer. Thirty-Eighth Annual CTRCAACR San Antonio Breast Cancer Symposium; 2015 Dec 8-12; San Antonio, USA. 